\title{
Itinéraires Itinéraires
}

Littérature, textes, cultures

2015-1 | 2015

Récits de société

\section{Style et idéologie dans le roman noir}

\section{Véronique Desnain}

\section{(2) OpenEdition}

Journals

Édition électronique

URL : http://journals.openedition.org/itineraires/2685

DOI : $10.4000 /$ itineraires.2685

ISSN : 2427-920X

Éditeur

Pléiade

\section{Référence électronique}

Véronique Desnain, «Style et idéologie dans le roman noir », Itinéraires [En ligne], 2015-1 | 2015, mis en ligne le 18 décembre 2015, consulté le 10 décembre 2020. URL : http://journals.openedition.org/ itineraires/2685; DOI : https://doi.org/10.4000/itineraires.2685

Ce document a été généré automatiquement le 10 décembre 2020.

\section{(c) (i) (9)}

Itinéraires est mis à disposition selon les termes de la licence Creative Commons Attribution - Pas d'Utilisation Commerciale - Pas de Modification 4.0 International. 


\title{
Style et idéologie dans le roman noir
}

\author{
Véronique Desnain
}

L'intrigue n'est que le squelette du roman noir, sa chair, c'est l'histoire sociale. (Pons 1997 : 7)

\section{Du roman de détection au roman noir}

1 Si le roman de détection classique (c'est-à-dire celui dans lequel la résolution d'une énigme est présentée comme la fonction principale du texte) cache bien son positionnement idéologique, le roman noir et sa version française post-soixante-huitarde, le néo-polar, mettent bien au contraire l'énigme au second plan, au profit d'une réflexion sur les origines même du crime, qu'ils placent fermement dans un contexte social et politique. Mon principal objectif dans cet article sera d'analyser la façon dont la volonté affirmée d'auteurs tels que Daeninckx et Manotti, en passant par Manchette, d'insuffler à leurs récits une dimension idéologique visible influence l'évolution du genre et la forme même du récit. Si de nombreux critiques (Vareille 1986, Kemp 2005 et 2006, pour n'en citer que deux) se sont attachés à démontrer une préoccupation partagée entre néo-polar et nouveau roman quant à la recherche d'une nouvelle écriture, il s'agira plutôt ici de considérer les éléments stylistiques et structuraux qui émergent directement, et peutêtre inévitablement, du désir des auteurs de mettre en lumière la dimension sociale de leurs textes.

2 Il convient tout d'abord de noter que le roman policier en général ne peut être qu'un reflet de la société puisque, même dans sa dimension la plus ludique, il présente un crime et les motivations possibles de chaque personnage et que la crédibilité du récit dépend d'une perception commune à l'auteur et aux lecteurs de ce qui est socialement acceptable. De plus il nous faut bien reconnaître que la définition de ce qui constitue un acte répréhensible est en grande partie dépendante du discours social dominant.

3 Le roman policier, de par sa nature même, met donc en scène une rupture : le moment où l'ordre social supposé correct est remis en question par l'irruption de la violence, où le chaos menace. De ce fait, comme le fait remarquer Kracauer, le roman policier « présente 
à la société déréalisée sa propre face, sous une forme plus pure qu'elle ne pourrait la voir autrement. Ses représentants et ses fonctions rendent ici compte d'eux-mêmes et trahissent leur signification cachée » (Kracauer [1971] 2001 : 52). Par conséquent, il serait naïf de supposer que le roman policier traditionnel, d'énigme ou de mystère, n'a aucune portée idéologique. Comme le signale Kermode, le traitement du matériel herméneutique implique la provision d'éléments à partir desquels nous pouvons déduire un système idéologique ${ }^{1}$. Ceci ne suggère pas nécessairement que cette démarche soit consciente de la part de l'auteur bien qu'il puisse être porté, comme le remarque Gallix (1996:14), à « inclure des conceptions sociales, politiques, voire racistes dans la composition de ses personnages afin de justifier leur éventuelle culpabilité, ce qui entraîne une lecture idéologique ». On peut cependant affirmer que, dans le roman policier traditionnel, cette « inévitabilité idéologique » n'a pas pour but de remettre en question l'ordre politique et social qu'il met à contribution.

4 Si l'on peut attribuer une spécificité commune à ces romans, c'est sans doute leur fonction cathartique : dans sa forme la plus traditionnelle, le récit se veut rassurant et le lecteur peut s'attendre à un retour à l'ordre, c'est-à-dire à l'identification et à l'expulsion de l'élément perturbateur représenté par le criminel. Le crime appartient donc à l'individu, qui est présenté dans ce contexte comme une anomalie et son crime comme un dysfonctionnement ou un échec personnel. Cela se manifeste dans la forme par un récit linéaire, dans lequel la mise en évidence progressive d'indices matériels mène à un dévoilement final qui clôt le récit. Todorov note cependant que le roman d'énigme est basé sur un double récit : l'histoire du crime (que le criminel essaiera de cacher et qui le restera jusqu'à la conclusion), et l'histoire de l'enquête, qui forme la base du récit. « Il s'agit donc, » dit-il «dans le roman à énigme, de deux histoires dont l'une est absente mais réelle, l'autre présente mais insignifiante » (Todorov $1978: 13$ ). On peut en conclure que la portée idéologique du roman traditionnel est, soit inconsciente, soit sciemment "cachée", sa fonction didactique étant masquée par une focalisation ludique sur l'« histoire insignifiante».

5 Les auteurs du roman policier engagé, de Manchette à Daeninckx ou Manotti proposent au contraire de mettre en lumière les dysfonctionnements de la société, tels qu'ils sont perçus au prisme de leurs propres positionnements idéologiques - le terme «moral», utilisé par Manchette (1978) est sur ce point révélateur -, plutôt que de mettre au centre du récit un individu qui serait présenté comme un élément autonome et pervers. Pas de serial killer psychopathe, donc, au cœur de l'intrigue, mais des assassins professionnels et des agents de la corruption systémique. Dans la forme la plus politisée du roman policier, l'intrigue joue un rôle minime, justice est rarement faite, et il faut chercher ailleurs que dans la confirmation rassurante de l'ordre établi les motivations à la fois de l'auteur et du lecteur.

6 Manchette lui-même théorise l'existence de deux grands genres distincts dans le roman policier : «Si la loi n'est pas remise en cause, si elle est considérée comme une chose absolument bonne telle quelle, comme la règle de l'ordre, le délit alors doit être et est uniquement traité comme un désordre qu'il faut réparer » (Manchette $1997: 59$ ). On peut sans peine reconnaître ici la base même du roman à énigme « classique » dont nous avons parlé plus haut. «L'autre façon de prendre les choses », nous dit Manchette :

[...] c'est de ne pas considérer comme bonnes les conditions d'application de la loi. On est amené alors à relativiser la loi, à l'historiciser, à faire en quelque sorte une philosophie du Droit, et à noter que la loi est l'expression d'un rapport de forces sociales, dans une situation historique donnée. (Ibid. : 60) 


\section{Idéologie et roman noir}

7 L'intention des auteurs change donc, mais c'est cependant bien en vertu de son statut de « récit de société » que le roman policier attire les écrivains engagés. Le premier à en faire cas est encore une fois Manchette, chez qui la volonté politique dicte le choix du genre : le roman policier, littérature de "classe » mais aussi produit de consommation qui a pour but de distraire plutôt que de donner à réfléchir, est à la fois le médium et la cible idéaux pour le projet marxiste et situationniste de Manchette, puisque :

[...] le genre se conforme à l'esprit du capitalisme libéral en exprimant et véhiculant une certaine conception de la productivité. La formule du roman d'enquête introduit sur le marché un type de récit voué à une production-consommation aussi rapide qu'efficace. (Dubois $1992: 26$ )

8 Ainsi «l'histoire du roman policier est une histoire sociale, car elle apparaît comme inextricablement liée à l'histoire de la société bourgeoise - voire de la production marchande - et surdéterminée par elle » (Mandel 1986). Manchette va donc s'attacher à démontrer que le crime n'est pas un fait isolé et relevant d'un dysfonctionnement de l'individu mais une conséquence inévitable de l'organisation sociale, économique et politique, et à attaquer la société capitaliste en se servant de ses propres réseaux de production et de distribution. Ainsi, pour lui, «[1]e bon roman noir est un roman social, un roman de critique sociale, qui prend pour anecdote des histoires de crimes, mais qui essaie de donner un portrait de la société » (Manchette [1993] 1996: 6).

Bien sûr, ce " portrait » ne peut en aucun cas être compris comme un récit objectif. Il est au contraire écrit pour dénoncer l'idéologie dominante et, comme nous allons le voir, il signale clairement sa subjectivité. Il répond ainsi également à ce que certains auteurs perçoivent comme un manque de regard critique de "l'autre » littérature. Il n'est pas rare en effet pour les auteurs de polar de se situer, non pas par rapport aux romans policiers traditionnels, mais par rapport à la littérature dite "blanche». Comme l'explique Vautrin (1997: 131) : «Je trouve assez que le roman noir, à l'envers de nos nombrils de Français bien nourris, continue à porter les germes d'une critique sociale comme il n'en existe à aucun étage de notre littérature en col blanc. » À la littérature du « moi », doit donc s'opposer une littérature de la société, et, celle-ci ne peut être que le polar puisque comme l'affirme le critique marxiste Ernest Mandel (1986) : "L'assassin c'est le système. » Et, dans son chapitre sur « Le "nouveau polar" français de l'après-68 ", il justifie cette affirmation:

Mais peu importe l'étiquette, il s'agit de saisir la tendance générale, la nature du genre, celle d'une mise en question radicale de la société dans son ensemble, de l'État et de ses appareils, y compris de la police, y compris des détectives privés. La violence, qui est toujours la caractéristique principale du genre, n'est plus avant tout criminelle et individuelle, ni exceptionnelle, comme dans le roman d'espionnage. C'est la violence institutionnelle quotidienne - ou, si l'on veut, le terrorisme d'État - qui est catégoriquement dénoncée, à laquelle s'oppose l'insignifiante mini-violence des laissés-pour-compte. (Mandel 1986 : n. p.)

Cette violence n'est pas seulement institutionnelle, elle est aussi inhérente à l'idéologie capitaliste qui privilégie avant tout le profit et conçoit donc l'individu comme un simple rouage dans cette mécanique de production. Ce n'est donc pas par hasard que le style de Manchette (qui a par ailleurs traduit Westlake et Bloch parmi d'autres) s'inspire d'auteurs américains, qui écrivent au cœur même du pays où ce monde de pensée est roi : 
La particularité de Hammett et de quelques-uns de ses contemporains c'est de faire servir le style behavioriste ${ }^{2}$ à une démythification sociale. Ce ne sont plus seulement les psychologies des individus qui sont " cachées dans le livre »; ce sont les rapports sociaux ; c'est le mensonge social qui maintient l'ordre. (Lafarge, cité dans Frommer $2001: 89$ )

11 De fait, le « crime » n'est plus révélé par des indices scientifiques (à la Sherlock Holmes) ou par la connaissance de l'âme humaine et le pouvoir d'observation du détective (comme chez Poirot ou Maigret), quand bien même il existe encore un mystère. La part de détection est souvent réduite au minimum dans ces romans, où le détective n'est plus que très rarement l'avatar du lecteur, qui loin de résoudre une énigme, suit fréquemment le déroulement du crime " en direct ». La surprise de la révélation finale est remplacée par le suspense et par la nécessité de comprendre les causes profondes de l'apparition même du crime. Le processus de dévoilement du premier récit selon Todorov (le crime) est donc remplacé par la suggestion d'une vérité plus large qui concerne non plus un incident spécifique mais une "situation» qui génère (et continuera de générer) ce type d'incidents. Ou comme le dit Manotti (2007: 107): «Dans l'histoire policière "traditionnelle", le crime est un acte individuel, qui renvoie à des mobiles personnels, des relations interpersonnelles [...] Le roman "noir", lui, enracine les crimes dans les circonstances sociales dans lesquelles ils sont commis. » Mandel va plus loin et présente le roman policier comme un produit même du cadre idéologique :

La société bourgeoise, née de la violence, la reproduit constamment et en est saturée. Elle provient du crime et conduit au crime, commis à une échelle de plus en plus industrielle. En définitive, l'essor du roman policier s'explique peut-être par le fait que la société bourgeoise, considérée dans son ensemble, est une société criminelle. (Mandel 1986 : n. p.)

12 On assiste donc à un renversement du schéma de Todorov. Dans ce renversement, le crime, en tant qu'action unique et individuelle, devient insignifiant, simple prétexte, alors que l'enquête, elle, donne sa signification au récit. Alors que dans le récit traditionnel les indices sociaux et idéologiques ont une fonction utile mais limitée, qui est de révéler des comportements "déviants» qui permettront au détective (et donc au lecteur) d'identifier le criminel, dans le polar engagé, ces mêmes indices servent à révéler des failles institutionnelles ou des vérités masquées par les discours officiels et les communications de l'idéologie dominante, qui est elle-même révélée comme néfaste. La démarche herméneutique n'est plus seulement liée à l'intrigue policière mais au déchiffrage/décodage du fonctionnement de la société tel qu'il n'est pas habituellement visible au citoyen lambda, c'est-à-dire au lecteur. Comme le soulignent Müller et Ruoff $(2002: 17)$ :

Comme pour la théorie critique, la société est, pour le polar, un scandale. Comme la théorie critique, il trouve les causes des crimes actuels dans la structure et l'histoire de la société bourgeoise et, comme elle, il prend pour hypothèse que la réconciliation ne saurait être obtenue par la seule élucidation des faits.

13 Cette préoccupation politique avouée des auteurs post-Mai 68 conduit donc à une transformation de la structure même de toute une partie du roman policier, ainsi qu'à l'émergence de caractéristiques liées au style.

Ainsi, le schéma traditionnel d'un récit linéaire, au cours duquel la révélation progressive d'indices matériels mène à un dévoilement basé sur la logique et qui met fin à la curiosité, et donc à l'intérêt du lecteur, va à l'encontre des intentions de l'auteur engagé. Ce schéma donne en effet à voir des personnages souvent caricaturaux et donc peu réalistes, des 
motifs purement personnels et le rétablissement d'un ordre présenté comme «naturel » et bénéfique. Le roman noir va avoir recours à un certain nombre de stratégies pour contester cette vision du monde.

\section{Effets de style : le narrateur politisé chez Manchette}

Manchette ouvre la voie en 1971 avec L'Affaire N'Gustro, basé sur l'enlèvement de Mehdi Ben Barka en $1965^{3}$. Par le biais d'Henri Butron, antihéros fictionnel, et des circonstances qui l'amèneront à être impliqué dans l'affaire "N'Gustro ", Manchette dévoile les liens entre la politique et la finance. Ce mélange de fiction et de réalité, ou plutôt cette exposition de la réalité au travers de la fiction, se retrouve très clairement chez Daeninckx et chez Manotti, qui poussent d'ailleurs le concept plus loin et créent un « effet de réel » à deux niveaux : d'une part en basant leurs textes sur des événements connus et nommés en tant que tels (les manifestations d'Octobre 1961 et la déportation des Juifs pendant l'occupation dans Meurtres pour mémoire pour Daeninckx, l'affaire Daewoo dans Lorraine Connection pour Manotti, par exemple), d'autre part en incorporant à leurs textes des documents tels que titres et articles de journaux, dépêches AFP ou statistiques authentiques, liés à ces événements.

Un peu plus tard, Le Petit Bleu de la côte Ouest (1976), qui est lui entièrement fictionnel, introduit un certain nombre d'éléments stylistiques que l'on va par la suite retrouver dans de nombreux romans policiers. L'auteur signale fréquemment sa subjectivité par l'incorporation de divers effets de style. D'abord, une écriture behavioriste qui, par sa focalisation externe et son caractère purement factuel, suggère clairement que les motivations individuelles et les mobiles personnels sont insignifiants. Le narratif à la troisième personne n'offre au lecteur qu'une perspective très limitée sur les raisons qui poussent les personnages à l'action ou sur leurs sentiments face aux événements dans lesquels ils sont impliqués. Si le narrateur offre occasionnellement un accès limité aux pensées d'un personnage, ces interventions sont spéculatives et ne semblent être là que pour renforcer le portrait antipathique d'un protagoniste que la société de consommation a vidé de tout sentiment compassionnel : ayant découvert un homme blessé sur le bord de la route, Gerfaut le fait monter dans sa voiture pour le transporter à l'hôpital, ce faisant « il pensait que le sang allait souiller le cuir des sièges; ou bien il ne pensait rien » (Manchette $1976: 23$ ).

17 Ce récit à la troisième personne qui, loin d'être neutre, porte manifestement un jugement négatif sur le protagoniste, inclut également un vocabulaire idéologiquement marqué :

La raison pour laquelle George file ainsi sur le périphérique avec des réflexes diminués et en écoutant cette musique-là, il faut la chercher surtout dans les rapports de production. (Manchette $1976: 8$ )

Il n'y a pas moyen de dire avec précision comment ça va tourner, les choses, pour Georges Gerfaut. Dans l'ensemble, on voit comment ça va tourner, mais avec précision on ne voit pas. Dans l'ensemble, ils vont être détruits, les rapports de production dans lesquels il faut chercher la raison pour laquelle Georges file ainsi sur le périphérique avec des réflexes diminués en écoutant cette musique-là. Peut-être Georges manifestera-t-il alors autre chose que la patience et la servilité qu'il a toujours manifestées. Ce n'est pas probable. Une fois, dans un contexte douteux, il a vécu une aventure mouvementée et saignante; et ensuite tout ce qu'il a trouvé à faire, c'est rentrer au bercail. Et maintenant, au bercail, il attend. Le fait qu'avec son bercail George tourne à $145 \mathrm{~km} / \mathrm{h}$ autour de Paris indique seulement que George est de son temps et de son espace. (Ibid. : 184) 
Il semble probable que la façon peu subtile dont Manchette manipule la terminologie marxiste ait en partie pour but d'exposer l'idéologie inhérente à tout récit, y compris les romans policiers de quelque nature qu'ils soient. Cette spécificité sémantique s'accompagne par ailleurs d'une focalisation sur le matériel (marques, fétichisation des objets) qui nous rappelle l'engagement situationniste et anticonsumériste de l'auteur. La nature circulaire du récit et son manque de clôture sont également frappants, puisque le roman s'ouvre et se ferme sur deux phrases, et sur deux actions, similaires :

George Gerfaut est en train de rouler sur le boulevard périphérique extérieur. Il est deux heures et demie ou peut-être trois heures un quart du matin [...] Georges Gerfaut roule à $145 \mathrm{~km} / \mathrm{h}[. .$.$] un lecteur de cassettes diffuse à bas niveau du jazz de$ style West-Coast. (Manchette 1976 : 7-8)

Gerfaut a rejoint le boulevard périphérique extérieur à la porte d'Ivry. En ce moment, il est $2 \mathrm{~h} 30$ ou peut-être $3 \mathrm{~h} 15$ du matin et Gerfaut tourne autour de Paris à $145 \mathrm{~km} / \mathrm{h}$ en écoutant de la musique West Coast... (Ibid. : 183-184)

Ces éléments apparaissent de nouveau dans La Position du tueur couché en 1982, dans lequel Martin Terrier quitte sa ville natale pour devenir tueur à gages afin de faire fortune et de revenir épouser la jeune femme qui lui a été refusée. Victime de la classe bourgeoise, qui refuse de voir l'une des leurs épouser un "pauvre", Martin est rendu brièvement ambitieux (du moins dans un contexte capitaliste) par les circonstances et se construit une belle carrière de tueur à gages qui lui permettra de s'enrichir avant de revenir conquérir celle qu'il aime. Mais on n'échappe pas si facilement à la fatalité moderne et Martin, dépouillé de sa fortune vient reprendre la place laissée vacante par son père dans l'ordre social. La nature circulaire du récit est renforcée par le fait que père et fils gardent la trace de la violence infligée par un système qui place le profit avant tout : le père avait miraculeusement survécu à une tentative de meurtre par ses concurrents ferrailleurs, Martin, lui, échappe à une tentative de meurtre par ses anciens employeurs qui n'apprécient pas son désir de prendre sa retraite. Le parallélisme des destins du père et du fils, et en particulier la façon dont les jeunes clients bourgeois s'amusent d'eux, suggère un déterminisme social auquel nul ne peut échapper :

Charles Terrier a reçu une balle de fusil Mauser dans la tête, où elle est restée et lui provoque parfois des sortes de crises, surtout s'il a bu [...] Charles Terrier s'est remis à boire depuis quelque temps. Des copains de Martin, des gars de la petite bande lui ont offert le coup à la brasserie où Charles est serveur, et la perturbation qui en est résultée vite dans le comportement de l'employé les a amusés beaucoup. (Manchette [1982] 1998: 52)

Au reste le serveur de brasserie [Martin Terrier] est normal. Il fait convenablement son travail, bien qu'il ait parfois de la maladresse motrice. Récemment on a observé que cette maladresse grandit s'il a bu des alcools. Les fins de soirée, quelques jeunes gens s'amusent parfois de lui offrir à boire, de sorte que l'homme se livre à des excentricités. (Manchette [1982] 1998 : 195)

Il convient de noter ici que si l'écriture de Manchette signale clairement son engagement politique, son long "silence » littéraire entre La Position du tueur couché et La Princesse du sang (entamé en 1989 et resté infini à sa mort en 1995) ne l'est pas moins. Comme le note Doug Headline dans sa préface de La Princesse du sang, Manchette perçoit le polar des années 1980 comme une "récupération" des mouvements sociaux des décades précédentes, un objet de consommation " parfaitement intégré à l'ordre des choses » et qui a donc perdu sa vocation subversive ${ }^{4}$. Qui plus est, il perçoit La Position du tueur couché comme un apogée puisque ce texte atteint le «style comportementaliste total» (Ibid.) qu'il recherchait. Il se concentre donc sur d'autres formes d'écritures (celle de scénarii en 
particulier). Pourtant, cela ne signifie pas que Manchette abandonne définitivement le polar. Il part au contraire à la recherche d'une autre façon de continuer sa démarche littéraire et militante. Attentif aux remous politiques, il comprend qu'une nouvelle forme de roman noir doit répondre au nouvel ordre politique, un ordre qui est justement en train d'émerger de la globalisation liée à l'idéologie capitaliste. Ainsi La Princesse du sang aurait été le premier volume d'un cycle qui mélangerait «roman noir et histoire politique », qui offrirait « une toile de fond à l'échelle mondiale et non plus nationale, le déploiement de forces occultes qui manipulent un grand nombre de pions » (Headline 1996 : 13). Loin donc de représenter un rejet du roman noir ou un abandon de son engagement politique en littérature, le silence de Manchette semble au contraire avoir été une période de réflexion nécessaire à l'élaboration d'une nouvelle approche qui mêle plus étroitement que jamais l'analyse idéologique à un style dépouillé, factuel : l'individu est ainsi représenté comme un simple rouage dans un vaste système dont il ne saisit ni l'ampleur ni la réelle influence. Si Manchette n'eut pas l'opportunité de mener ce projet à sa conclusion, son influence ne s'en fait pas moins sentir dans le roman noir français de la fin $\mathrm{du} \mathrm{xx} \mathrm{e}^{\mathrm{e}}$ siècle.

\section{De l'engagement politique à la littérature engagée}

21 Le détachement qui caractérise l'écriture behavioriste de Manchette se retrouve chez Manotti sous la forme d'une écriture sèche, qui privilégie le dialogue dans des chapitres courts, tous introduits par une date (lundi 3 mars, mardi 4 mars, etc.) et qui donne au récit un ton factuel et documentaire. Cette impression est d'ailleurs renforcée par l'insertion, à divers endroits du roman, de coupures de presse, statistiques et autres documents authentiques. Cependant, l'objectivité du récit à la troisième personne, remise en cause par l'utilisation d'un vocabulaire connoté dans Manchette, est également dénoncée comme un leurre dans Sombre Sentier par l'apparition de la première personne du singulier dans l'un des derniers paragraphes du roman : « Finalement, on n'a pas fait la fête, pour célébrer notre victoire. C'est le seul regret que j'aie gardé de ce printemps 1980 » (Manotti 1995 : 313). Cette intrusion soudaine de la première personne du singulier recadre le récit dans la perspective de l'auteur-témoin, qui nous rappelle en dernière instance que certains événements du récit sont réels, même si les personnages ne le sont pas. De même, la raison pour laquelle « on n'a pas fait la fête » est que «[...] quand la régularisation a été bien engagée, le coup d'État venait d'avoir lieu, en Turquie. Et chacun était obsédé par la violence de la répression, là-bas » (Ibid.). La résolution du récit est donc remise en question par l'allusion à de nouveaux désordres.

D'ailleurs l'épilogue, intitulé "Quinze ans après ", nous signale que les principaux coupables, c'est-à-dire non pas ceux qui ont littéralement tué, mais les responsables de toute la filière criminelle de prostitution et de drogue à la base de l'intrigue, et dont la protection était assurée par leurs clients haut placés dans le gouvernement et la finance, ces coupables donc, « vivent aux Bahamas, où ils s'ennuient » (Manotti 1995 : 313). Pas de rétribution donc pour ces criminels, qui font partie intégrante du système, et pas de catharsis rassurante pour le lecteur.

Enfin, le récit se clôt, tout comme il s'était ouvert, sur un paragraphe de statistiques sur le marché de la drogue, nous rappelant ainsi que les crimes individuels auxquels nous avons assisté dans l'intrigue ne sont que les conséquences d'un problème beaucoup plus global que la mise à l'écart ou l'élimination de quelques individus ne suffira pas à résoudre. Cette 
triple absence de clôture peut donc être perçue comme la "preuve» que le mal est systémique plutôt qu'individuel. L'angoisse que peut générer cette conclusion est encore intensifiée par la dimension internationale du récit. Comme l'avait prédit Manchette, cette nouvelle incarnation du roman noir reflète la difficulté à circonscrire le crime dans un contexte où celui-ci ne peut plus être perçu comme un simple acte délinquant et la responsabilité du seul individu. La globalisation implique de vastes réseaux qui placent le profit avant toute chose et dans lesquels la frontière entre transactions légales et criminelles est souvent floue. La notion de souveraineté nationale est de plus en plus menacée par les intérêts économiques globaux et la notion de crime comme phénomène local que les lois locales sont à même de réprimer semble de plus en plus dépassée. Qui plus est, il apparaît clairement que les nombreux agents, économiques autant qu'idéologiques puisque les deux vont inévitablement de pair, qui jouent un rôle difficilement discernable dans des réseaux étroitement liés à des activités criminelles à grande échelle, sont eux-mêmes liés au pouvoir.

Cette problématique est également mise en scène chez Daeninckx. Même si l'œuvre de celui-ci se concentre plus largement sur la France, le désir de révéler les structures cachées du pouvoir constitue l'objectif principal de ses romans. Comme celui de Manotti, qui revendique son passé marxiste et qui fut longtemps impliquée dans des activités syndicales, l'engagement politique de Didier Daeninckx est connu : longtemps membre du parti communiste, il reste fermement de gauche et ces textes portent un regard critique à la fois sur l'histoire (la Seconde Guerre mondiale, la guerre d'Algérie, la colonisation) et sur les problèmes contemporains qui en découlent (négationnisme, racisme, politique d'immigration). Ainsi, ses romans vont mêler événements réels et personnages fictionnels pour remettre en question la version officielle de certains épisodes historiques afin d'exposer leurs liens avec le contexte présent et de contester un discours politique qui prétend à la vérité tout en manipulant l'information. Cette stratégie s'est révélée particulièrement efficace dans Meurtres pour mémoire (1984) qui a donné lieu à une réévaluation extensive des événements d'Octobre $1961^{5}$. Plusieurs des éléments structuraux et stylistiques déjà mentionnés se retrouvent chez Daeninckx : utilisation de documents historiques (titres et extraits d'articles de journaux, statistiques, affiches) ainsi que de dates et de lieux précis et surtout des dénouements qui mettent en avant l'impossibilité pour l'individu de combattre seul un système néfaste et corrompu, qui protège les criminels dès lors que ceux-ci servent l'idéologie dominante: Veillut, le "criminel d'état», collaborateur devenu fonctionnaire haut placé de Meurtres pour mémoire est certes assassiné mais par l'un de ses anciens subordonnés, empêchant ainsi toute justice officielle. Qui plus est, l'assassin présente son geste à l'inspecteur Cadin, qui s'apprêtait à confronter Veillut, comme un acte de protection envers le policier : « il vous aurait eu, petit... C'était joué d'avance " (Daeninckx 1984 : 205), suggérant ainsi que les pouvoirs de la police, même si ses membres sont intègres, sont pour le moins limités face à la nécessité pour les politiques de créer ce que Cadin nomme à la fin du roman « un scénario plus conforme à l'idée que les citoyens devraient se faire des garants de l'ordre public» (Ibid.: 213). Une possibilité qui semble par ailleurs entérinée par la mort du commissaire Londrin dans la conclusion de Lumière noire : ayant persisté dans son enquête malgré les conseils de ses supérieurs, Londrin est abattu par les services spéciaux chargés d'éliminer les témoins d'une bavure policière. De plus, les scrupules de Londrin font figure d'exception parmi les personnages de policiers présentés par l'auteur. L'exemple le plus frappant de l'image d'une police au service du pouvoir plutôt qu'à celui de la justice apparaît dans Itinéraire d'un salaud ordinaire (2006), dans lequel Clément Duprest traverse 
quarante ans d'histoire, de la rafle du Vél' d'Hiv jusqu'à l'élection présidentielle de 1981, en fonctionnaire "apolitique» et irréprochable. Suivant les ordres en toute bonne conscience, même lorsque cela implique de véritables crimes contre l'humanité. Duprest est emblématique de la méfiance envers les forces de l'ordre qui émerge de l'œuvre de Daeninckx. Cette méfiance s'accompagne d'un pessimisme rendu évident par le destin de l'inspecteur Cadin, qui, bien que parfois complice du système (il accepte de ne pas rendre public les résultats de son enquête dans Meurtres pour mémoire), vit malgré tout fort mal son rôle au sein de l'appareil policier. Ce malaise culmine dans Le Facteur fatal (1990), un recueil de nouvelles qui retrace les enquêtes, et bien souvent les échecs, de Cadin, inspecteur dérangeant muté de poste en poste (chaque chapitre a lieu dans une ville différente) de 1977 à 1989, avant de conclure sur le suicide de l'inspecteur :

$52 \ldots 53 \ldots 54 \ldots$ À 55 il posa le canon contre sa tempe. À 56 son index droit entra en contact avec la détente. Il respira lourdement, une dernière fois, et appuya, les yeux grands ouverts au moment exact où les chiffres 23h59mn59s s'affichaient pour être remplacés par une série de zéros. Dehors on se mettait à klaxonner, les fenêtres s'ouvraient sur les cris de joie, les baisers, les chants. Le monde entrait dans les années 1990. (Daeninckx 1990 : 200-201)

La date est sans aucun doute significative et le suicide symbolique: il marque non seulement la fin de l'inspecteur Cadin, mais aussi la désillusion des auteurs de l'après 68, engagés dans la littérature en partie parce que déçus par le militantisme pratique et par la « gauche Mitterrand ${ }^{6} »$. Ainsi comme le remarquent les éditeurs du dossier consacré au polar de la revue Mouvements en 2001 :

C'est au moment où les sociétés françaises et italiennes se dépolitisent que le polar prend une teinte de plus en plus critique et tend à se "politiser». Étrange paradoxe, dont la compréhension repose peut-être sur la rencontre entre une génération désillusionnée, en rupture avec l'engagement politique traditionnel, mais toujours attachée intellectuellement à une vision sombre et critique de la société, et des auteurs issus eux-mêmes de la gauche et pris pour la plupart dans cette tension [...] [Les classes moyennes urbaines cultivées] y ont trouvé un moyen " confortable", mais aussi individuel et distancié d'autres formes d'engagement politique, de maintenir un regard critique sur la société. (Corcuff et al. $2001: 6$ )

Une théorie qui semble confirmée par Manotti à la fois dans sa fiction et dans son analyse de son travail d'écrivain, lorsqu'elle déclare avoir commencé à écrire des romans noirs au moment même où elle a arrêté son activisme politique, un activisme qui «n'allait nulle part » (Manotti, cité dans Belhadjin 2010 : 74).

Elle a récemment illustré ce propos au travers de la fiction dans L'Évasion, paru en 2013 et qui raconte le passage à l'écriture de Fillipo, prisonnier de droit commun en Italie et évadé en compagnie de Carlo, un détenu politique. À Paris, en 1987, parmi les réfugiés politiques, il fait le récit de son évasion et de son amitié avec Carlo et connaît un bref succès littéraire avant d'être abattu en pleine rue, sans doute par les services secrets italiens qui craignent qu'il n'en sache plus qu'il ne le dit. Comme à son habitude, Manotti fait allusion dans ce texte, à travers des personnages fictionnels, à des événements réels, ici les « années de plomb » italiennes. Mais ce n'est pas là le seul point de contact avec la réalité et il est difficile de lire ce texte sans faire un lien avec l'affaire Battisti : membre des Prolétaires armés pour le communisme, Cesare Battisti est arrêté et condamné en 1979 pour appartenance à une bande armée. Il s'évade en 1981 et se réfugie au Mexique, où il commence à écrire. En 1982 cependant, il est accusé par la justice italienne d'avoir été impliqué dans quatre meurtres. En 1990, sous la protection de la «doctrine Mitterrand ${ }^{7}$ ", il s'installe à Paris et entame une carrière d'écrivain de polar, dont les 
textes sont bien reçus par la critique. En 2004, l'Italie renouvelle sa demande d'extradition, qui est acceptée par le gouvernement français, de droite à cette époque, et Battisti est arrêté. Cette décision, perçue par de nombreuses personnalités de gauche comme une trahison de l'État français, donne naissance à un mouvement de soutien dont la figure de proue sera une autre " polardeuse », Fred Vargas ${ }^{8}$.

Bien que Manotti ne mentionne jamais directement cette affaire, les parallèles entre fiction et réalité sont parlant : Battisti, comme Fillipo fut détenu de droit commun et s'est politisé en prison, il fut gardien d'immeuble, Fillipo est gardien de nuit, tous deux sont à Paris en vertu de la « doctrine Mitterrand ». Qui plus est, Carlo est abattu lors d'un holdup par un tireur d'élite de la police qui attendait dans la banque. Lisa, ancienne compagne de Carlo et militante d'extrême gauche, elle aussi réfugiée à Paris, est convaincue que le hold-up up a en fait été organisé par les services secrets italiens et que Carlo est tombé dans un piège destiné à discréditer toute l'extrême gauche. Il est impossible de ne pas faire le lien ici avec l'attentat de la Piazza Fontana à Milan en 1969 (auquel le roman fait d'ailleurs référence): l'attentat est à l'époque attribué officiellement aux anarchistes, quatre-vingts personnes sont arrêtées, dont une meurt dans des conditions suspectes durant sa garde à vue. L'enquête révélera bien des années plus tard que l'attentat était sans doute le fait d'une organisation néofasciste, mais aussi que les services secrets italiens et la CIA auraient participé à cette action en fournissant les explosifs. Les motifs proposés dans L'Évasion pour l'implication des gouvernements dans de telles opérations sont liés non seulement à leur positionnement idéologique ("la peur du rouge ») mais également à la détermination des gouvernants de protéger leur propre position. Comme le dit l'un des terroristes présumés : « nous sommes les grands méchants qui occupons les premiers rôles, et donc les tribunaux, les unes des journaux. Et derrière, on escamote leurs turpitudes à eux » (Manotti 2013 : 192).

De tels exemples forment la base du discours des défenseurs de Battisti qui affirment que les accusations dont il est l'objet furent exagérées ou fabriquées afin de le discréditer politiquement. On pourrait donc ici voir Fillipo et Carlo comme des personnages hybrides, représentant les deux versants (auteur et activiste) de Battisti.

Mais ce qui ressort comme le sujet principal du texte est le passage de l'action (représentée par Carlo, et par les souvenirs confiés à Fillipo) à l'écriture, et surtout leur aptitude respective à révéler la vérité. La position de Manotti semble tout à fait claire lorsqu'elle conclut L'Évasion par ces mots, prononcés par Lisa, qui a jusqu'alors tenté d'exposer les manipulations et les mensonges des services secrets italiens durant les « années de plomb »: «Oui, j'abandonne. Ce combat-là est perdu. Si je veux essayer de sauver notre passé, il ne me reste plus qu'une chose à faire. Écrire des romans » (Manotti 2013 : 211).

31 Ainsi c'est cette volonté de refléter, mais aussi de dénoncer qui explique à la fois le choix du genre et les mutations que lui font subir les auteurs mentionnés ici. Dans le passage de l'action à la fiction, la vision militante impose un nouveau modèle d'écriture qui répond à des demandes bien différentes de celle du roman policier traditionnel. C'est le désir de rendre explicite l'idéologie implicite du roman policier qui le fait passer d'une structure linéaire, close et monophonique, à une structure beaucoup plus complexe, mettant en avant la subjectivité de voix narrative, souvent circulaire, ou en tout cas peu satisfaisante 
du point de vue du récit de détection, et dans laquelle les signes linguistiques ou stylistiques signalent volontairement la vocation politique du texte.

\section{BIBLIOGRAPHIE}

Belhadjin, Anissa, 2010, «From politics to the roman noir », South Central Review, $\mathrm{n}^{\circ}$ 27, p. 61-81.

Collovald, Annie, 2001, «L'enchantement dans la désillusion politique », Mouvements, $\mathrm{n}^{\circ}$ 15-16, p. 16-21.

Corcuff, Philippe, et al., 2001, « Le polar entre critique sociale et désenchantement », Mouvements, $\mathrm{n}^{\circ}$ 15-16, p. 5-7.

Dubois, Jacques, 1992, Le Roman policier ou la Modernité, Paris, Nathan.

Forsdick, Charles, 2001, « "Direction les oubliettes de l'histoire”: witnessing the past in the contemporary French polar », French Cultural Studies, n 12 , p. 333-350.

Gallix, François, 1996, « Formes du roman policier de détection : quelques approches de la critique moderne ", dans W. Dow (dir.), Formes et structures génériques du roman policier, Paris, Presses de l'université Paris-Sorbonne, p. 11-25.

Headline, Doug, 1996, dans La Princesse du sang, Paris, Gallimard.

Kemp, Simon, 2005, « Les pièces à Conviction: Perception in French Crime Fiction and its Literary Influence », Modern and Contemporary France, vol. 13, n 2, p. 149-60.

-, 2006, Defective Inspectors: Crime-Fiction Pastiche in Late-Twentieth-Century French Literature, Oxford, Legenda.

Kermode, Frank, 1983, « Novel and Narrative », dans G. W. Most et W. W. Stowe (dir.), The Poetics of Murder: Detective Fiction and Literary Theory, New York, Harcourt, p. 175-196.

Kracauer, Siegfried, 2001, Le Roman policier [1971], Paris, Payot et Rivages.

Lafarge, 2001, cité dans Frank Frommer, « Jean-Patrick Manchette : le facteur fatal », Mouvements, $\mathrm{n}^{\circ} 15-16$, p. 88-95.

Manchette, Jean-Patrick, 1978, « Le polar est la grande littérature morale de notre époque », Charlie Mensuel, $\mathrm{n}^{\circ} 108$.

-, 1997, « Note sur le roman policier », Polar « Spécial Manchette », p. 59-60.

-, entretien sur France Culture, 1993, cité dans la préface de La Princesse du sang, Paris, Payot \& Rivages, 1996.

Mandel, Ernest, 1986, Meurtres exquis : histoire sociale du roman policier, Paris, La Brèche, [Extrait, en ligne], http://www.ernestmandel.org/new/ecrits/article/l-assassin-c-est-le-systeme-le, consulté le 7 janvier 2014.

Manotti, Dominique, 2007, « Roman noir », Le Mouvement social, n² 219-220, p. 107-109.

Müller, Elfriede et Ruoff, Alexander, 2002, Le Polar français : crime et histoire, Paris, La Fabrique. 
Pons, Jean, 1997, « Le roman noir, littérature réelle », Les Temps modernes, n 595, p. 5-14.

Todorov, Tzvetan, 1978, « Typologie du roman policier », dans Poétique de la prose, Paris, Seuil, p. 56-57.

Vareille, Jean-Claude, 1986, « Culture savante et culture populaire : brèves remarques à propos des horizons idéologiques, des structures et de la littéralité du roman policier », Caliban, $\mathrm{n}^{\circ} 23$, p. 5-19.

Vautrin, Jean, 1997, «Le roman noir, espace libre et enragé », Les Temps modernes, nº 595, p. 130-136.

\section{Corpus de l'étude}

Daeninckx, Didier, 1984, Meurtres pour mémoire, Paris, Gallimard.

-, 1990, Le Facteur fatal, Paris, Denoël.

-, 2006, Itinéraire d'un salaud ordinaire, Paris, Gallimard.

Manchette, Jean-Patrick, 1976, Le Petit Bleu de la côte Ouest, Paris, Gallimard.

-, [1982] 1998, La Position du tueur couché, Paris, Gallimard.

-, [1971] 1999, L'Affaire N'Gustro, Paris, Denoël.

Manotti, Dominique, 1995, Sombre sentier, Paris, Seuil.

-, 2013, L'Évasion, Paris, Gallimard.

\section{NOTES}

1. Kermode (1983: 184) : «The processing of hermeneutic material has entailed the provision of material from which we may infer an ideological system. "

2. Le terme "behavioriste", est utilisé ici et plus loin dans cet article pour décrire un style d'écriture qui s'attache à une description neutre des actions, sans explication d'ordre psychologique permettant au lecteur d'identifier les motivations des personnages.

3. Mehdi Ben Barka était un opposant au régime marocain et fondateur de L'Union nationale des forces populaires. Exilé en France en 1963, il fut kidnappé par des policiers français en 1965, et sans doute exécuté. Le ministre de l'Intérieur marocain, Mohammed Oufkir fut accusé d'avoir été impliqué dans cette disparition. Dans L'Affaire N'Gustro, Henri Butron organise un rendez-vous fatal entre N'Gustro, le chef de l'opposition et Oufiri, le ministre de l'Intérieur d'un pays africain fictionnel.

4. Propos recueillis dans Libération en 1991et cités par Doug Headline (1996: 10).

5. Sur ce sujet, voir par exemple Charles Forsdick (2001).

6. Manchette et Daeninckx furent tous les deux actifs en mai 68, Manotti était auparavant responsable syndicale. Le titre de son roman Nos fantastiques années fric (2001) est une référence au premier septennat de Mitterrand. Voir aussi Annie Collovald (2001).

7. En 1985, Mitterrand s'engage à ne pas extrader les anciens activistes italiens ayant rompu avec la violence, à l'exclusion des crimes de sang. Accusé de meurtres, Cesare Battisti n'est pas à proprement parlé protégé par cette doctrine, mais dans les faits il ne sera jamais inquiété.

8. Parmi ceux qui soutiennent Battisti, et l'affirment innocent des crimes dont il est accusé, figurent également Georges Moustaki, Bertrand Delanoë, Bernard-Henri Lévy et l'Abbé Pierre. Son innocence (ou sa culpabilité) n'a pour l'instant pas été établie. En 2002, Battisti se réfugie au 
Brésil. Le gouvernement brésilien a jusqu'à présent refusé son extradition, au motif que son retour en Italie pourrait mettre en danger son intégrité physique.

\section{RÉSUMÉS}

Cet article a pour but d'examiner la façon dont le positionnement idéologique des auteurs de roman noir de la fin $\mathrm{du} \mathrm{xx}^{\mathrm{e}}$ siècle se manifeste dans les aspects stylistiques et structuraux du texte. En se basant en particulier sur les textes de Jean-Patrick Manchette, Didier Daeninckx et Dominique Manotti, il démontrera que le passage à l'écriture de ces auteurs ne peut être dissocié de leurs expériences politiques et militantes et que les mutations observables dans le polar à partir des années 1980 est une conséquence directe de leur désir de rendre explicite l'idéologie implicite dans toute œuvre littéraire, et dans le roman policier en particulier.

This article aims to examine the way in which the ideological posture of late twentieth century crime fiction writers impacts on stylistic and structural aspects of the text. Taking novels by Jean-Patrick Manchette, Didier Daeninckx and Dominique Manotti as examples for this analysis, the extent to which these authors' writing is influenced by their experience as political activists will be highlighted. It will also show that the changes that can be observed in French crime fiction from the 1980s to the present are a direct consequence of their desire to make explicit the ideology implicit in all works of fiction, and in particular in crime fiction.

\section{INDEX}

Mots-clés : crime, fiction, idéologie, histoire, style

Keywords : crime fiction, noir fiction, ideology, history

\section{AUTEUR}

\section{VÉRONIQUE DESNAIN}

The University of Edinburgh 